\title{
High Throughput Correlation of Dendritic Spines: 2-photon in vivo live imaging to SEM Utilizing the Automated Tape-Collecting Ultramicrotome and Array Tomography
}

\author{
Connon Thomas ${ }^{1}$, Naomi Kamasawa ${ }^{1}$, Shani Peter ${ }^{1}$, Kuo-Sheng Lee $^{2}$ and David Fitzpatrick ${ }^{2}$ \\ 1. Electron Microscopy Core Facility, Max Planck Florida Institute for Neuroscience, Jupiter, FL, USA \\ 2. Department of Functional Architecture and Development of Cerebral Cortex, Max Planck Florida \\ Institute for Neuroscience, Jupiter, FL, USA
}

Recently, in vivo imaging techniques using light microscopy have been used as a powerful tool to study synaptic interactions and neural circuits in the brain. However, without visualizing structural details using electron microscopy (EM), it is difficult to confidently identify whether two "crossing" processes are synapsing or passing by coincidence. Correlation traditionally involved TEM imaging of serial sections, but this process is difficult, prone to errors, and time consuming. Therefore, we developed a workflow designed for high throughput imaging by exploiting the Automated Tape Collecting Ultra-Microtome (ATUM) to semi-automatically collect thousands of ultrathin sections. Combining this with Atlas 5, an array tomography software interface used to acquire and stitch a large dataset of SEM images, we previously showed it was possible to analyze spinogenesis from reasonably large volumes in cultured brain slices [1]. Here, we expand the application to correlate in vivo 2-photon live imaging with SEM array tomography of pyramidal neurons in layer $2 / 3$ of the tree shrew visual cortex.

Spines of pyramidal neurons expressing GCaMP6 were examined for their orientation tuned responses to visual stimuli in vivo. Animals were then perfused and brain tissue was sliced at $50 \mu \mathrm{m}$. Target cells were found using epifluorescence microscopy, and a high resolution Z-stack of the same area was imaged using confocal laser scanning microscopy. The tissue was then processed for EM and immunolabeled with nanogold (postsynaptic) and biotin (presynaptic) conjugated antibodies, followed by silver enhancing and a DAB reaction, respectively. Correlating in the xy plane was accomplished by comparing blood vessel patterns to those in the epifluorescence images [2]. The tissue was trimmed to a size of ca. $1 \times 1.5 \mathrm{~mm}$, sectioned at $50 \mathrm{~nm}$ using the ATUMtome (RMC/Boeckeler), and placed onto silicon wafers. Wafers were coated with a thin layer of carbon $(5 \mathrm{~nm})$ and imaged using a Merlin VP Compact and Gemini SEM300 (Carl Zeiss).

First, low resolution images $(1 \mu \mathrm{m} / \mathrm{pixel})$ were captured to find the target labeled cell, again using blood vessels as a reference to the light microscope images. Then, the approximate depth of target dendritic segments was identified by comparing the confocal $\mathrm{z}$ stack to the position of ultrathin sections on the wafer, as both optical section thickness and ultrathin section thickness was known. A rough reconstruction of the cell from this depth was then performed using higher resolution images ( $30 \mathrm{~nm} / \mathrm{pixel}$ ), which served as a "3D map" to the segment of dendrite that contained target spines. This segment was then imaged at highest resolution $(6 \mathrm{~nm} / \mathrm{pixel})$ and spines were correlated to data collected during functional imaging. Morphological characteristics such as PSD length, vesicle organization, and presynaptic input to spines could then be correlated to in vivo functional responses. By optimizing imaging conditions, this process could be completed in a matter of weeks, rather than the months or years it would take using traditional TEM serial sections. 
This method allows for a direct and more efficient comparison of functional properties of spines at the level of light microscopy to their ultrastructural elements using the electron microscope [3].

\section{References:}

[1] N Kamasawa et al, Microscopy and Microanalysis 21 (2015), p. 1271.

[2] P De Boer, JP Hoogenboom and BNG Giepmans, Nature methods 12(6) (2015), p. 503.

[3] The authors thank the specialists of Applications and Services from RMC/Boeckeler, Fibics, and Carl Zeiss Microscopy for their insensitive support.
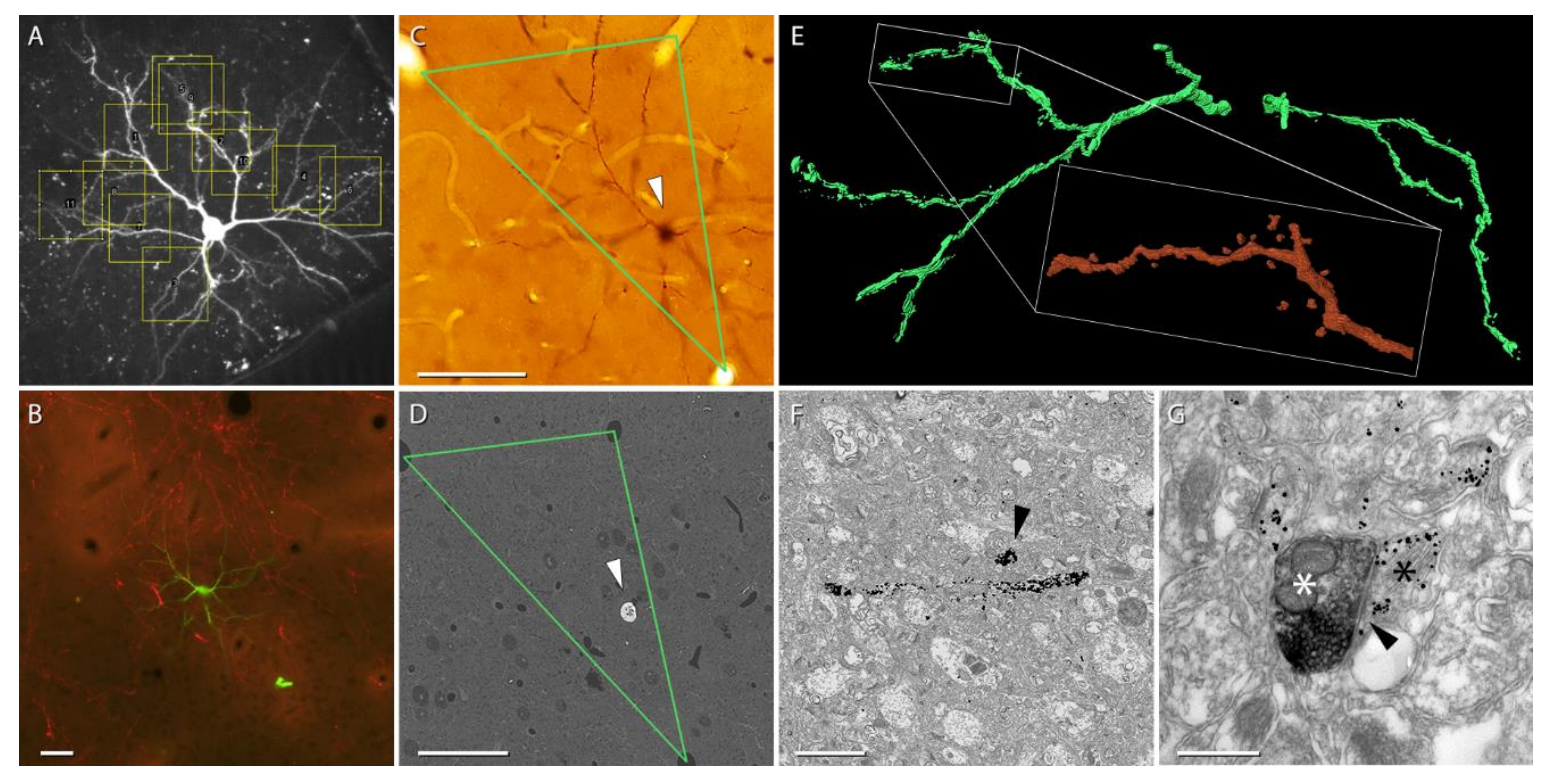

Figure 1. Various stages of the correlative imaging workflow. (A) 2-photon microscope image of a GCaMP6 labeled layer 2/3 pyramidal neuron, with regions of interest (yellow boxes). (B) Epifluorescence image of the same cell depicted in (A) showing sparse GCaMP6 labeling (green) and RFP labeled layer 4 axons (red). (C) and (D) Predicting the location of the cell soma (arrows) in the xy plane for SEM relocalization using blood vessel markers. (E) Rough partial reconstruction (green) of the dendritic field of the neuron, used to identify the locations of targeted dendritic segments for high resolution reconstruction (orange). (F) SEM micrograph of an anti-GFP gold labeled dendrite and spine (arrow). (G) A synapse between a DAB labeled presynaptic terminal (white asterisk) and gold labeled spine (black asterisk). Note that vesicles are still visible after dense DAB labeling. Arrow denotes postsynaptic active zone. Scale bars: $50 \mu \mathrm{m}(\mathrm{B}-\mathrm{D}), 2 \mu \mathrm{m}(\mathrm{F}), 500 \mathrm{~nm}(\mathrm{G})$. 